


\title{
La parodia como marco estructural en Don Quijote y Los viajes de Gulliver
}

\section{Parody as the structural frame on Don Quixote and Gulliver's travels}

\author{
Alfonso Muñoz Corcuera' \\ Universidad Nacional Autónoma de México
}

\begin{abstract}
Resumen
Los viajes de Gulliver, de Jonathan Swift, es una de las obras más conocidas de la literatura universal. Sin embargo, su recepción actual, al menos en Espańa, se produce sobre todo por medio de adaptaciones en las que no se puede apreciar el calado de la obra literaria original. En este estudio se pretende profundizar en el análisis de Los viajes de Gulliver a través de una comparación con Don Quijote, enfocándose en los recursos paródicos: mientras Don Quijote parodia los libros de caballerías, Los viajes de Gulliver parodia los libros de viajes. El principal objetivo de esta comparación no será tanto demostrar una influencia de la obra de Cervantes sobre la de Swift, sino, más bien, iluminar algunos aspectos de Los viajes de Gulliver.
\end{abstract}

${ }^{1}$ Agradezco sus comentarios sobre versiones previas de este texto a Tatiana Bubnova Gulaya, Rocío Peñalta Catalán y Elisa Di Biase Castro. Becario del Programa de Becas Posdoctorales en la UNAM, Instituto de Investigaciones Filológicas. 
Palabras clave: Don Quijote, Gulliver, parodia, literatura inglesa, literatura española.

\section{Abstract}

Gulliver's Travels, of Jonathan Swift, is one of the most well-known works in world literature. However, its actual reception, at least in Spain, it's produced mostly through adaptations in which it's impossible to appreciate the profundity of the original literary work. In this article, I intend to dig deeply into the analysis of Gulliver's Travels through a comparison with Don Quixote, focusing on the parodic resources: while Don Quixote parodies the books of chivalry, Gulliver's Travels parodies travel books. The main goal of this comparison will not be to prove some influence of Cervantes' work in Swift's work, but to illuminate some aspects of Gulliver's Travels.

Keywords: Don Quixote, Gulliver, Parody, English literature, Spanish literature.

\section{Introducción}

Cituar una obra literaria cualquiera en disposición de ser $\checkmark$ analizada desde una perspectiva crítica es siempre una cuestión problemática. Más aún cuando se trata de realizar una comparación. Agreguemos que si para dicha comparación el crítico se aventura a seleccionar dos obras no sólo de distinta época, sino también de distinta procedencia e incluso de distinto género, y además estas obras son dos de las cumbres de la literatura universal (como es el caso que aquí nos ocupa), bien haríamos en seguir un consejo que Sancho Panza podría darnos: las comparaciones son odiosas y en boca cerrada no entran moscas.

A pesar de estas objeciones, pues la sabiduría popular no deja de tener sus razones en el siglo XXI, presento en las siguientes páginas una comparación entre la historia de El Ingenioso Hidalgo Don 
Quijote de la Mancha, según la dio a conocer al mundo Miguel de Cervantes en 1605 y 1615, y los no menos conocidos Viajes a varias naciones remotas del mundo, que si bien fueron publicados como escritos por Lemuel Gulliver en 1726, resultaron ser redactados por Jonathan Swift, motivo por el cual son conocidos finalmente bajo el nombre de Los Viajes de Gulliver. En esta comparación no trataré de buscar qué obra hace mejor o peor su función, o si una es hija de la otra, lo que nos conduciría a los problemas comunes en toda comparación. Sino que, atendiendo al consejo popular, trataré prudentemente de mostrar en qué sentido Los Viajes de Gulliver, sin haber nacido necesariamente de una influencia directa de la obra de Cervantes, mantiene un espíritu cervantino en ciertos aspectos, especialmente al presentarse como una parodia de un libro de viajes, al igual que Don Quijote se presentaba como una parodia de un libro de caballerías.

Para ello, partiré de un estudio de la presencia de la obra cervantina en el siglo XVIII en las Islas Británicas y de la lectura de ésta que pudo hacer el párroco irlandés, así como del contexto literario de ambos autores. Posteriormente, me centraré en el análisis de ambos textos como parodias de sendos géneros literarios en boga en la época en que fueron publicadas las dos obras -los libros de caballerías a principios del siglo XVII en España y los libros de viajes a principios del siglo XVIII en las Islas Británicas-, señalando los paralelismos que se pueden detectar entre la obra cervantina y la de Swift.

Se me podría objetar que, al ser mi trabajo una comparación entre dos obras (donde la primera fue leída y admirada por el autor de la segunda, y existiendo evidentes paralelismos entre las mismas), no se defienda la filiación directa de la segunda con respecto a la primera por simple recato. Sin embargo, tal y como se verá a lo largo de este estudio, los motivos que llevaron a ambos autores a realizar sus obras son bien distintos, lo que se traduce en 
que las obras poseen notables diferencias a pesar de los paralelismos que guardan. Por otro lado, los posibles indicios textuales que pudieran permitir la defensa de una influencia directa no son lo suficientemente claros como para poder aseverar que Swift se inspiró de un modo global en Don Quijote para la composición de Los Viajes de Gulliver, aunque sí se inspiró en la obra de Cervantes para la composición de algún pasaje concreto; en este sentido, el presente artículo se puede considerar programático; también porque la obra de Swift comparte con la de Cervantes un marco estructural basado en la parodia de sendos géneros literarios, por eso es pertinente investigar si dicha influencia se extiende al propio marco estructural de la obra. El análisis que aquí se presenta sólo apunta hacia los lugares en que podría detectarse dicha influencia, sin defender ni negar su existencia. En cualquier caso, e independientemente de si existe la influencia (cuestión propia de otro tipo de trabajo), la comparación que realizaré resultará valiosa por sí misma, pues iluminará ciertos aspectos clave de la obra de Swift.

Una vez aclarado este punto, sólo resta dar por concluida esta introducción y entrar definitivamente en materia. Sin embargo, no quisiera cerrar este exordio sin antes dar algún indicio, por vago que sea, de ese espíritu cervantino que noto en Los Viajes de Gulliver, de modo que al verlo el lector se convenza de que hay algo cierto en mi tesis y se anime definitivamente a seguir adelante con esta lectura. Y no se me ocurre mejor manera de ver cumplido mi deseo que traer a colación un fragmento de un discurso de don Quijote que, de alguna extraña forma, nos hace pensar que está hablando de su lectura de Los Viajes de Gulliver sin ser ello posible, a no ser a modo de profecía:

Ya no hay [caballero] ninguno que saliendo deste bosque entre en aquella montaña, y de allí pise una estéril y desierta playa del mar, las más veces proceloso y alterado, y hallando en ella y en su orilla 
un pequeño batel sin remos, vela, mástil ni jarcia alguna, con intrépido corazón se arroje en él, entregándose a las implacables olas del mar profundo, que ya le suben al cielo y ya le bajan al abismo, y él, puesto el pecho a la incontrastable borrasca, cuando menos se cata, se halla tres mil y más leguas distante del lugar donde se embarcó, y saltando en tierra remota y no conocida, le suceden cosas dignas de estar escritas, no en pergaminos, sino en bronces (Cervantes Saavedra, 1998: 633-634).

\section{Don Quijote en las Islas Británicas en el siglo XVIII}

La novela de Cervantes tuvo desde un momento muy temprano una gran acogida entre el público inglés (por ejemplo, vid. Paulson, 1997; Randall y Boswell, 2009; Garrido Ardila, 2014). No en vano, apenas tres años después de su publicación en España el dramaturgo inglés Francis Beaumont estrenó su obra Knight of the Burning Pestle, en la que se recrean la locura y las aventuras de Don Quijote en un aprendiz de tendero londinense. Ese mismo año, Thomas Middleton, también autor de teatro, evocó el episodio de los molinos de viento en su Your Fair Gallants (Medina Casado, 2005: 432-433). Otra muestra de esta rápida recepción, es que poco tiempo después se publicó en las Islas Británicas la primera traducción de Don Quijote a una lengua extranjera, obra de Thomas Shelton en 1612, la cual en 1620 fue reeditada, cuando se hizo la primera traducción a una lengua extranjera de la segunda parte, también a cargo de Shelton (Medina Casado, 2005: 411). ${ }^{2}$

La popularidad de Don Quijote en las Islas Británicas no paró de crecer durante todo el siglo XVII. Su influencia, en un primer momento, se hizo notar sobre todo en el teatro y en la poesía, cuestión que no deja de ser sorprendente por el trasvase genérico

${ }^{2}$ Aunque la atribución de la autoría de la traducción de la segunda parte es discutida (vid. Randall y Boswell, 2009: XXI). 
que supone. Sin embargo, esto tiene su explicación en el hecho de que no fue hasta finales del siglo XVI que la novela inglesa comenzó a desarrollarse, con la evolución de los antiguos romances medievales, por lo que a principios del siglo XVII todavía se encontraba en un estado demasiado germinal. La literatura espańola, una de las más prestigiosas de la época, tuvo gran importancia en este proceso, como se puede comprobar a través del estudio de la influencia de obras como La Celestina, Lazarillo de Tormes o Guzmán de Alfarache. No obstante, sobre todas ellas destacará la influencia de Don Quijote, pues gracias su imitación se deberá el considerable desarrollo de la novela moderna inglesa a lo largo de todo el siglo XVIII (Hammond, 1998). Autores como Daniel Defoe (1660-1725), Jonathan Swift (1667-1745), Samuel Richardson (1689-1761), Henry Fielding (1707-1754) o Laurence Sterne (1713-1768), todos ellos grandes admiradores de la obra cervantina, fueron los principales responsables de este desarrollo, sin el cual, todo sea dicho, posiblemente la influencia de Don Quijote en la literatura posterior no habría sido la misma. ${ }^{3}$

Tradicionalmente se ha considerado a Fielding el heredero más directo del legado cervantino (Pardo García, 1995-1997: 133); por eso con él, a menudo, se inicia el estudio de las relaciones entre Cervantes y la novela inglesa (Pardo García, 2005). Ya en 1734 había estrenado una ópera que llevaba por título Don Quixote in England, clara muestra de su admiración por las aventuras del hidalgo castellano. Sin embargo, fue gracias a sus novelas que adquirió la fama de heredero del escritor complutense. El título de su primera gran obra, The History of the Adventures of Joseph Andrews, and of his Friend Mr. Abraham Adams. Written in Imitation of the Manner of Cervantes, Author of Don Quixote (1742),

${ }^{3}$ Apunto esto porque la reinterpretación que estos autores hicieron de la obra de Cervantes fue adoptada posteriormente por novelistas y críticos del resto de Europa (vid. Close, 1998: CXLIX). 
habla por sí mismo. El escritor inglés toma prestado del español el estilo humorístico, la tendencia al realismo, la introducción de episodios e historias paralelas a la trama principal, algunas escenas de confusión que se suceden durante la noche en una posada y su excelente percepción de lo ridículo en personajes y acciones, ${ }^{4}$ además de la importancia del papel del narrador y de las reflexiones metaliterarias en la propia estructura de la obra (Medina Casado, 2005: 444).

\subsection{Swift, lector de Cervantes}

En este contexto literario, no resulta extraño afirmar que Jonathan Swift era, como la mayoría de sus contemporáneos, un gran admirador de Miguel de Cervantes (Elena, 2003: 53). Tanto de su correspondencia como de sus trabajos en prosa, se pueden extraer abundantes referencias a Cervantes o a Don Quijote (Guerra de Gloss, 1988: 213-214), y entre sus obras de ficción se pueden rastrear varios pasajes que sin duda fueron moldeados con la obra del escritor español como referente. Entre estas últimas, especial atención ha recibido a este respecto la obra satírica Cuento de una barrica (1704), la primera publicación de importancia de Swift. La obra ha sido señalada por algunos críticos como dependiente del modelo de Don Quijote, aunque este aspecto no es aceptado de forma unánime (Medina Casado, 2005: 440-441). Lo que sí es evidente son las tres ocasiones en que se cita de modo más o menos explícito la novela española, la primera de ellas en una de las piezas preliminares que de una forma muy cervantina se presenta como un aviso del librero al lector pero que la crítica coincide en atribuir al propio Swift; también, han sido estudiados los paralelismos entre la locura de Jack -uno de los personajes protagonistas de

${ }^{4}$ Esto último lo destaca el propio Fielding en el prefacio de la novela. 
Cuento de una barrica- y la de don Quijote, así como el uso de la ironía que hacen ambos autores en sus respectivas obras (vid. Guerra de Gloss, 1988).

En cuanto a Los Viajes de Gulliver, su obra más conocida y también la que aquí nos ocupa, se han realizado pequeños estudios comparando, por ejemplo, el carácter de Gulliver y don Quijote, en cuanto a su credulidad (Ernst, 2005), o los distintos puntos de vista que tienen los personajes, en cuanto a su relación con la realidad al final de sus peripecias (Domínguez de Paz y Carrascosa Miguel, 1990). Sin embargo, no deja de ser significativo que ambos trabajos, de pequeña extensión, señalen que todavía queda por hacer un estudio más profundo de las relaciones entre Don Quijote y la obra más importante del párroco irlandés.

Finalmente, se han señalado algunas coincidencias entre ambos autores en relación a algunas obras menores de Swift, como por ejemplo algunos de sus poemas escatológicos o La batalla entre los libros antiguos y modernos. En los primeros se ha señalado, además del carácter quijotesco de algunos de sus personajes, la influencia de la ironía cervantina en el estilo swiftiano (Guerra Bosch, 1999). En cuanto a La batalla entre los libros antiguos y modernos, podría relacionarse de forma tangencial con Don Quijote atendiendo al modo en que ambos autores tratan la literatura de su tiempo (Domínguez de Paz y Carrascosa Miguel, 1990; también, vid. Bando Domínguez, 2007; Torralbo Callejero y Gámez Fernández, 2007).

Un hecho curioso, que no quiero dejar de señalar, es que una de las múltiples traducciones de Don Quijote realizadas a lo largo del siglo XVIII corrió a cargo de Charles Jarvis (o Jervas), pintor de origen irlandés y amigo de Jonathan Swift (Medina Casado, 2005: 422-423). Sin embargo, a pesar de ser una de las más populares de todo el siglo XVIII, su traducción no vería la luz hasta 1742, casi veinte años después de la publicación de Los Viajes 
de Gulliver. A pesar de ello, aunque la traducción de Jarvis podría haber estado terminada en 1725 (Hammond, 2009: 97), un año antes de la publicación de la obra de Swift, no parece probable que dicha traducción influyese en la obra que nos ocupa. Teresa Guerra señala que posiblemente Swift leyera Don Quijote a partir de la traducción de John Phillips de 1687 (Guerra Bosch, 1999: 121), la cual es bastante libre pues incluso modifica en algunas partes el texto original, motivo por el que no fue muy popular en su época. Por su parte, A. C. Elias señala que hacia mediados de la década de 1720 Swift pudo iniciar su propio proyecto de traducción de la obra de Cervantes (Elias, 1998). En cualquier caso estas eventualidades no afectan a nuestro estudio de modo sustancial.

\section{Los libros de caballerías y los libros de viajes en el momento de la aparición de Don Quijote y Los Viajes de Gulliver}

Las dos obras que nos ocupan en este trabajo poseen en común el hecho de ser parodias de unos géneros literarios concretos. Por ese motivo, antes de empezar a compararlas, creo que será útil saber en qué situación estaban este tipo de libros-ambos prácticamente abandonados en la actualidad- en el momento en que se publicaron las obras de Cervantes y Swift respectivamente, de modo que se puedan comprender mejor las relaciones que estableció cada una con el género que estaba parodiando y los objetivos que movieron a cada autor a realizar su crítica. Para ello, primero me referiré a los libros de caballerías y a su importancia dentro de Don Quijote, para posteriormente analizar la relación entre Los Viajes de Gulliver y los libros de viajes. 


\subsection{Los libros de caballerías \\ en la España del Siglo de Oro}

Tradicionalmente se ha considerado que los ataques de Cervantes a los libros de caballerías en Don Quijote fueron el motivo de la pérdida de popularidad de un género que gozaba de buena salud a principios del siglo XVII. Sin embargo, tal y como ha demostrado la crítica en los últimos años, esto no fue exactamente así. Los libros de caballerías habían iniciado ya un claro declive y la obra de Cervantes no tuvo una gran incidencia en su ya anunciado fin. No obstante, debido al prestigio que adquirió la obra de Cervantes, especialmente a partir del siglo XVIII, la recepción crítica de estos libros sí se vio muy condicionada por la parodia que de ellos hizo el autor de las Novelas Ejemplares, de modo que hasta bien entrado el siglo XX apenas han recibido atención por parte del mundo académico. Esta situación, afortunadamente, no se mantiene en la actualidad y los estudios sobre los libros de caballerías, especialmente en relación con Don Quijote, han sido bastante abundantes en los últimos ańos (vid. p. ej. Guijarro Ceballos, 2007; Williamson, 1991).

Dentro de la literatura caballeresca española se engloban un número cercano a los setenta títulos, todos ellos escritos en un abanico temporal que se mueve entre finales del siglo XIII -momento en que se introduce el género en la península- y principios del siglo XVII -el Policisne de Boecia, publicado en 1602, fue el último libro castellano original en salir a la luz-. Su popularidad durante el Siglo de Oro fue elevadísima, siendo, a pesar del descenso en su consideración a partir de 1600, el sector editorial más importante de toda la época áurea (Roubaud, 1998: CVIII-CIX).

El género gozó de una inmejorable salud durante la primera mitad del siglo XVI, pues era leído por un público amplio y 
variado, no sólo por una minoría selecta. Incluso miembros de las capas más bajas de la sociedad, generalmente analfabetos, tenían conocimiento de las aventuras que se contaban en estos libros a través de lecturas públicas, como se refleja en una de las escenas de la venta en la primera parte de El Quijote. Sin embargo, ya a partir de 1550 se nota un descenso considerable en el ritmo en el que se suceden las ediciones de las distintas obras, situación que se acentúa todavía más a partir de 1600 . Al mismo tiempo, las nuevas producciones van degenerando, exagerando cada vez más los tópicos, ya presentes en el Amadis de Gaula-ejemplar más importante del género-, hasta convertirlos en auténticos galimatías (Roubaud, 1998: CXV-CXVI). Con todos estos datos se puede apreciar sin dificultad que en el momento en que apareció Don Quijote los libros de caballerías eran un género en claro proceso de abandono.

En esta situación, es perfectamente comprensible la voluntad de Cervantes de escribir "una invectiva contra los libros de caballerías" (Cervantes Saavedra, 1998: 17), como anuncia en el prólogo de la primera parte. El género, a pesar de contar todavía con un buen número de lectores -como seguirá teniéndolos aún durante buena parte del siglo XVII-, era muy criticado por los intelectuales de la época. De hecho ya en el siglo XVI habían sido objeto de severas críticas, e incluso en 1555 las Cortes propusieron, sin éxito, su prohibición (Eisenberg, 1995). En este sentido, el mensaje de Cervantes podía ser fácilmente compartido por sus contemporáneos.

\subsection{Los libros de viajes en la Inglaterra del siglo XVIII}

Los libros de viajes, al igual que los libros de caballerías en la España del Siglo de Oro, fueron durante el siglo XVII y principios del XVIII uno de los géneros literarios más en boga en Inglaterra. 
Pero a diferencia de los anteriores, no se trataba de un género de ficción. Los libros de viajes recogían las experiencias de viajeros reales en tierras lejanas y contenían detalladas descripciones de sus habitantes, de su flora, de su fauna... El interés del público por conocer los territorios que se estaban descubriendo y colonizando hacía de este tipo de escritos uno de los más cotizados -recuérdese que es precisamente a principios del siglo XVII cuando Inglaterra comienza a colonizar Norteamérica-, ${ }^{5}$ motivo por el cual los relatos de viajes reales se leían con avidez y se les confería una gran importancia en todo tipo de discusiones, ya fuesen científicas o teológicas. Cientos de viajeros se dirigían a explorar nuevas tierras, con el fin de hallar nuevos datos para la ciencia incipiente y siguiendo pautas facilitadas por la Royal Society, ${ }^{6}$ y volvían con un material precioso, del que se beneficiaban tanto los científicos como los filósofos (García Tortosa, 1973: 35-36).

No obstante, como bien señala García Tortosa, las cosas cambiaron en el siglo XVIII y "los viajes imaginarios [de los que Los viajes de Gulliver es una muestra] son una continuación lógica de las narraciones de viajes reales" (García Tortosa, 1973: 15), consecuencia de la adaptación de los primeros a los cambios que había experimentado la sociedad. El impacto que la descripción

${ }^{5}$ Curiosamente, el interés por conocer el Nuevo Mundo estuvo unido en Inglaterra a un fuerte interés por conocer también el Viejo Mundo. Durante los siglos XVII y XVIII se popularizaron los viajes de formación por Europa, especialmente Italia, entre los jóvenes de la nobleza y la alta burguesía británica -lo que se conoce como el Grand Tour-, de tal modo que los libros de viajes ambientados en territorios inexplorados estuvieron acompañados de libros de viajes ambientados en la vieja Europa (vid. De Seta, 1996).

${ }^{6}$ Se ha de tener en cuenta que el siglo XVII es el momento en que despega definitivamente la ciencia moderna en Inglaterra. La Royal Society of London for Improving Natural Knowledge fue fundada en 1660, y Sir Isaac Newton, máximo exponente de este movimiento científico y cultural, vivió entre 1643 y 1727 , siendo presidente de la misma Royal Society desde 1703 hasta su muerte. 
de sociedades reales había hecho en la Inglaterra del XVII empezó a perder eficacia, puesto que el público cada vez estaba más familiarizado con aquellas costumbres extrañas (García Tortosa, 1973: 39), lo que provocó que paulatinamente las narraciones de viajes reales fuesen adquiriendo un aire novelesco, casi fantástico, con el que sus autores trataban de dotar de un mayor interés a sus escritos. De ahí que el lector del siglo XVII y principios del XVIII no supiese discernir, en ocasiones, entre un viaje imaginario y una narración de viaje real (García Tortosa, 1973: 35).

En este contexto, muy parecido al de los libros de caballerías en España a principios del siglo XVIII, resulta más sencillo entender los motivos que llevaron a Swift a parodiar los ya degenerados libros de viajes reales con un libro de viajes imaginarios. Con este objetivo, utilizó todos los recursos a su alcance para que sus Viajes a varias naciones remotas del mundo fuesen confundidos por el público con un auténtico libro de viajes. Así, además del pseudónimo que utiliza, Swift presentó a Lemuel Gulliver como un pariente del navegante inglés William Dampier, uno de los autores de libros de viajes más leídos de la época, tratando de dar así mayor verosimilitud a la historia que presentaba. Por otro lado, eligió una redacción con un estilo llano, sin apenas recursos literarios, y que presta atención a cada detalle que el personaje observa directamente, acorde con las instrucciones que la Royal Society daba a los verdaderos exploradores. ${ }^{7}$

En cualquier caso existe una clara diferencia entre la relación que se establece entre los libros de caballerías y Don Quijote y la que hay entre Los Viajes de Gulliver y los libros de viajes. Mientras la novela de Cervantes es una invención original en la literatura de

\footnotetext{
${ }^{7}$ Las instrucciones de la Royal Society se publicaron en 1692, además de en las actas de la propia sociedad entre 1665 y 1667, en un volumen separado bajo el nombre General Heads for a natural History of a Country... for the Use of Travellers and Navigators (Elena, 2003: 52-53).
} 
su tiempo, ${ }^{8}$ el libro de Swift se inscribe en su mayor parte dentro del marco de un género con una larga tradición en la literatura occidental, el de los viajes imaginarios -cuyo primer cultivador sería Luciano de Samosata-, y del que existen múltiples muestras en la literatura inglesa del siglo XVIII. ${ }^{9}$ Existe, en cualquier caso, un intenso debate acerca del género al que se debe adscribir Los Viajes de Gulliver, pues no es simplemente un libro de viajes imaginarios, sino que también posee características de la novela utópica, la sátira menipea, el cuento filosófico, la fábula moral o incluso la novela moderna de la que hablaba anteriormente, lo que muestra también la gran complejidad de esta obra (Elena, 2003: 58). Por otro lado, al participar de varios géneros, el componente de parodia de los libros de viajes presente en Los Viajes de Gulliver es menos significativo, desde mi punto de vista, que el presente en Don Quijote sobre los libros de caballerías.

\section{La parodia como marco estructural en Don Quijote y Los Viajes de Gulliver}

Una vez repasado el contexto literario en el que nos vamos a mover, ya estamos en disposición de comenzar con la comparación de estas dos obras. En mi plan de trabajo, en primera instancia,

${ }^{8}$ Aunque es pertinente señalar que la atribución de invención de un género nuevo posiblemente proviene más de las lecturas que se han hechos posteriormente de Don Quijote que de la intención de Cervantes. Como señala Eisenberg, Cervantes pudo haber concebido su libro como perteneciente al género de los libros de caballerías pese a su carácter burlesco con respecto a los mismos (Eisenberg, 1995: cap. 3).

${ }^{9}$ Muchas de ellas se escribieron debido a la influencia de Los Viajes de Gulliver, pues antes de un año de su publicación ya se habían salido dos continuaciones espurias; sin embargo, otras fueron publicadas incluso antes que la obra de Swift. Lo que sí parece original en Swift es la idea de parodiar los libros de viajes reales por medio de un libro de viajes imaginarios (vid. García Tortosa, 1973). 
pasaré revista a un fragmento que podría indicar una influencia de Don Quijote sobre la obra de Swift; posteriormente, analizaré las similitudes y las diferencias que existen entre las dos obras en el tipo de recursos que utilizan para parodiar los libros de caballerías y los libros de viajes respectivamente -lo que he llamado la parodia como marco estructural-, y para finalizar extraeré las conclusiones que sean pertinentes del análisis efectuado.

El principal motivo que podría inducirnos a considerar que Los Viajes de Gulliver están influidos por Don Quijote son las circunstancias históricas que rodean la obra de Swift. Como hemos visto, el irlandés era un gran admirador de Cervantes, al igual que la mayoría de sus contemporáneos; por lo que resulta difícil pensar que, en ese contexto, alguien proyectara realizar una parodia de un género literario sin tener en cuenta la más famosa parodia genérica de la historia de la literatura. Ésta es la intuición de fondo en el presente trabajo y el motivo por el que creo que Los Viajes de Gulliver poseen ese espíritu cervantino al que me he referido. En cualquier caso, antes de analizar los paralelismos entre las dos obras, me gustaría señalar una escena que apoya la tesis de que existe una influencia de Cervantes sobre Swift.

El fragmento al que me refiero es uno de los episodios recogidos en el tercer viaje de Gulliver, en el que el protagonista se encuentra visitando una academia de ciencias, la Academia de Lagado, y observa varios experimentos:

Iba quejándome de un ligero cólico y mi acompañante me llevó a una habitación donde se alojaba un eminente médico, famoso porque curaba esta dolencia con operaciones inversas de un mismo instrumento. Tenía un fuelle enorme con una boquilla larga y delgada de marfil. Ésta se introducía veinte centímetros ano arriba y, extrayendo aire, afirmaba, podía dejar las tripas tan huecas como una vejiga seca. Pero cuando el mal era más rebelde y violento, introducía la boquilla con el fuelle lleno de aire y lo 
descargaba en el cuerpo del paciente, extraía luego el instrumento para hincharlo de nuevo, mientras apretaba fuertemente con el pulgar el orificio del nalgatorio y, tras repetir esto tres o cuatro veces, el viento intruso se precipitaba fuera llevándose de paso lo malsano (como agua que se echa en una bomba) y el paciente se recuperaba. Le vi poner en práctica ambos experimentos con un perro, pero no pude advertir efecto alguno del primero. Después del segundo, el animal quedó para explotar y soltó una descarga lo bastante violenta para afectarnos muy seriamente a mí y a quienes me acompañaban. El perro murió en el acto y dejamos al doctor tratando de resucitarlo por el mismo procedimiento (Swift, 2003: 413-414).

La escena nos recuerda tremendamente a otra historia que Cervantes narra en el prólogo de la segunda parte de Don Quijote:

Había un loco en Sevilla que dio en el más gracioso disparate y tema que dio loco en el mundo, y fue que hizo un cañuto de caña puntiagudo en el fin, y en cogiendo algún perro en la calle, o en cualquiera otra parte, con el un pie le cogía el suyo, y el otro le alzaba con la mano, y como mejor podía le acomodaba el cańuto en la parte que, soplándole, le ponía redondo como una pelota; y en teniéndolo desta suerte, le daba dos palmaditas en la barriga y le soltaba, diciendo a los circunstantes, que siempre eran muchos: "¿Pensarán vuestras mercedes ahora que es poco trabajo hinchar un perro?» (Cervantes Saavedra, 1998: 619).

A este respecto podemos señalar que en una de las citas que hace Swift de Cervantes en sus trabajos en prosa -concretamente en una de las conocidas como cartas de Drapier, escritas entre 1724 y 1725, momento en el que Swift se encontraba componiendo Los Viajes de Gulliver (apud. Guerra de Gloss, 1988: 214)-10 el párroco

${ }^{10}$ Pilar Elena señala que el tercer viaje de Gulliver, en el que se encuentra la escena que estamos analizando, debió ser escrito entre 1725 y 1726 , dadas las 
irlandés se refiere a la otra historia del loco y el perro que aparece en el prólogo a la segunda parte de Don Quijote inmediatamente después del fragmento que aquí nos ocupa, lo que me hace pensar que Swift podría tener bastante presente esta historia de cuando escribió la escena que he transcrito. Lo que es más, como ya hemos señalado, a mediados de la década de 1720 Swift parece haber estado trabajando en su propia traducción de Don Quijote (Elias, 1998). Por eso, me atrevería a defender que existe una influencia directa de Don Quijote sobre Los Viajes de Gulliver que se cristaliza al menos en el fragmento citado. Es importante señalar que Pilar Elena, en su edición de Los Viajes de Gulliver, señala otras dos posibles fuentes para este pasaje, una literaria -Rabelais- y otra científica -prácticamente todo el tercer viaje es una sátira contra la ciencia de la época-, sin señalar la posible fuente cervantina (en nota al pie; Swift, 2003: 414). Sin embargo, además de los factores que ya hemos señalado, Swift había utilizado la imagen de hinchar a alguien con un fuelle por el ano en Cuento de una barrica para satirizar los discursos de los predicadores disidentes, hinchados artificialmente, del mismo modo que Cervantes utilizaba la historia del perro para atacar a Avellaneda por hinchar excesivamente la historia de don Quijote. Por lo que, teniendo en cuenta la reconocida influencia de la obra de Cervantes sobre Cuento de una barrica, me inclino a favorecer la hipótesis de que el fragmento referido de Los Viajes de Gulliver es una muestra de la influencia de Cervantes en la obra.

El fragmento del loco y el perro, unido a la admiración de Swift por Cervantes y a la huella de Don Quijote en el periodo de la vida de Swift en que éste se dedicó a componer Los Viajes de Gulliver, dejan claro que la influencia de Cervantes sobre la obra de Swift es más que probable. Sin embargo, a pesar de ello, no podemos

referencias históricas que en él se hallan (Elena, 2003: 96). 
olvidar que Los Viajes de Gulliver se inscriben dentro de un género concreto -el de los viajes imaginarios- con una cierta presencia en la Inglaterra de la época propiciada por la degeneración de los libros de viajes reales, hasta el punto de que a menudo era difícil distinguir entre ambos tipos de libros. En este sentido, sería perfectamente posible justificar la presencia de una parodia de los libros de viajes reales con un libro de viajes imaginarios sin necesidad de recurrir a ningún modelo externo. No obstante, a pesar de la posible influencia de Cervantes sobre Los Viajes de Gulliver, en el resto del artículo no pretendo ahondar en ella, pues hacerlo presupondría defender que Don Quijote es la base sobre la que Swift construye su obra, lo cual me parece demasiado aventurado. Por el contrario, me gustaría analizar ciertas similitudes y diferencias que existen entre las dos obras en el tipo de recursos que utilizan para parodiar. Mi propuesta puede entenderse en un sentido programático sobre aspectos que merecería la pena analizar más detenidamente en busca de una influencia más profunda de la obra de Cervantes. En cualquier caso, independientemente de que pudiese existir tal influencia, la comparación que realizaré resulta valiosa por sí misma, pues ilumina ciertos aspectos clave de la obra de Swift.

\subsection{El juego de autoría}

El primer recurso que me gustaría señalar es el juego que ambos autores entablan sobre la autoría de sus textos. Como bien es sabido, Cervantes se presenta como el simple recuperador de los manuscritos del historiador arábigo Cide Hamete Benengeli, del que habiendo encontrado unos cartapacios sobre don Quijote de la Mancha, los mandó traducir a una tercera persona, un morisco que acertaba a pasar por allí. El recurso es una parodia de uno de los tópicos en los libros de caballerías, pues también solían ser presentados como la traducción de un texto mucho más antiguo 
escrito por algún sabio en lengua extranjera; lo que permitía, por un lado, dotar de un aura de misterio y autenticidad a la obra y, por otro, que el autor real se diese a un juego de distanciamiento y perspectivas en relación con la narración (Marín Pina, 1998: 859). Cervantes aprovecha este tópico para hacer parecer que la historia es producto de un historiador arábigo, "siendo muy propio de los de aquella nación ser mentirosos" (Cervantes Saavedra, 1998: 110), traducida a su vez por un morisco, del que se podría decir otro tanto. Sin embargo, en el trasfondo de la parodia no se encuentra un deseo real de hacer pasar por cierta la historia de Cide Hamete Benengeli, pues el libro se publica como compuesto por Miguel de Cervantes Saavedra, como bien se puede leer en las portadas tanto de la primera edición de 1605 como de la segunda de 1615 .

En Los Viajes de Gulliver, por el contrario, el nombre de Jonathan Swift no aparece por ningún sitio. El libro se presenta como escrito por Lemuel Gulliver, "primero médico y luego capitán de varios barcos" (Swift, 2003: 170-171)," quien debido a sus escasas dotes literarias le entregó el manuscrito a Richard Sympson, un amigo que se encargó de hacerlo revisar por algunos "jóvenes caballeros de una u otra universidad" (Swift, 2003: 173) para finalmente darlo a la imprenta. La decisión de Swift de presentar su obra bajo un pseudónimo obedece a dos motivos distintos: por un lado, el libro presenta una fuerte sátira contra los más altos dirigentes de la época, especialmente contra el primer ministro de Inglaterra Robert Walpole, lo que podría hacer que Swift se viese perjudicado en caso de repercusiones; ${ }^{12}$ por otro, al ser Swift ya un personaje muy conocido, no podría haber firmado y presentado su obra como si fuese un auténtico libro de viajes, el empleo de un pseudónimo

${ }^{11}$ Traducción de lo incluido en la portada de la primera edición de 1726.

${ }^{12}$ Swift ya había visto perjudicada su carrera dentro de la Iglesia anglicana por uno de sus libros, Cuento de una barrica, que le hizo caer en desgracia ante la reina Ana (vid. Elena, 2003: 20-21). 
servía a su propósito de hacer pasar Los Viajes de Gulliver por un libro de viajes reales. En esta línea, Swift hace a Gulliver pariente y consejero de William Dampier, el autor más conocido de libros de viajes de la época -lo que no deja de tener un cierto parecido con los poemas laudatorios de la primera parte de Don Quijote-. Del mismo modo, en el prólogo a la segunda edición de la obra, Swift ańade una supuesta carta de Gulliver a Richard Sympson, acusándole de haber introducido modificaciones sustanciales en el texto original, de modo que en ocasiones le hace faltar a la verdad. De este modo, al sentirse indignado por estas supuestas mentiras, el resto de los pasajes parecen autentificados. Un detalle curioso es el apuntado por Pilar Elena a propósito del nombre del editor del texto, Richard Symspon, ya que Swift podría estar sugiriendo una conexión entre Los Viajes de Gulliver y la falsificación de un libro de viajes reales escrito por un tal Capitán William Sympson (es un pseudónimo) en 1715, A New Voyage to East-Indies, el cual estaba compuesto de pasajes copiados de otros libros de viajes reales (en nota al pie; Swift, 2003: 173).

\subsection{Los prólogos y las segundas ediciones}

Otro paralelismo, que está más relacionado con las circunstancias que rodearon la publicación de los libros que con las características de la parodia, es el papel que cumplen los prólogos en las distintas ediciones. Cervantes publicó las dos partes que componen Don Quijote por separado y con un lapso de diez ańos entre ambas, lo que condicionó en gran medida la forma en que se presentó la segunda parte, especialmente por haber sido publicada en el intervalo la continuación espuria de Alonso Fernández de Avellaneda a la que Cervantes se refiere explícitamente en su segundo prólogo.

Por su parte, Swift publicó Los Viajes de Gulliver de una sola vez. Sin embargo, al año siguiente se publicó una nueva versión 
en la que se corregían algunas erratas y se modificaban algunos fragmentos del texto que se supone habían sido introducidos por el primer editor para suavizar la sátira. En ese breve lapso de tiempo el libro se había convertido en un éxito, motivo por el cual se habían publicado ya varios libros de claves para interpretarlo, así como dos continuaciones espurias. Todo ello fue motivo para que Swift incluyese un nuevo prólogo en la segunda edición, el cual no deja de tener paralelismos con el prólogo a la segunda parte de Don Quijote, ya que ambos autores utilizan ese espacio para responder a las críticas que habían recibido sus obras en la primera edición.

\subsection{El lenguaje}

Una coincidencia más sutil es el uso del lenguaje como herramienta paródica. Si Cervantes ponía en boca de don Quijote la fabla caballeresca para parodiarla:

¡Oh princesa Dulcinea, señora desde cautivo corazón! Mucho agravio me habedes fecho en despedirme y reprocharme con el riguroso afincamiento de mandarme no parecer ante la vuestra fermosura. Plégaos, señora, de membraros desde vuestro sujeto corazón, que tantas cuitas por vuestro amor padece (Cervantes Saavedra, 1998: 47-48).

Swift decide renunciar a cualquier tipo de recurso literario que pudiese embellecer el discurso con el fin de imitar el supuesto objetivismo de los libros de viajes reales, prestando atención a cada detalle que el personaje observa directamente, acorde con las instrucciones que la Royal Society daba a los verdaderos exploradores:

Hecha la estima, nos encontramos a 30 grados 2 minutos de latitud sur. Doce tripulantes habían muerto del excesivo bregar y 
la mala comida, y los demás se encontraban muy débiles. El 5 de noviembre, que es cuando comienza el verano en aquellas regiones, y a través de una bruma espesa, los marineros pudieron percibir un escollo como a medio cable de la nave, pero el viento era tan fuerte que nos arrastró bruscamente hacia él, estrellándonos al instante. Seis miembros de la tripulación, yo entre ellos, lanzamos el bote al agua y a duras penas conseguimos alejarnos del navío y del escollo (Swift, 2003: 190-191).

De la misma forma, en ocasiones se enreda en complicadas descripciones de las maniobras náuticas que realizan en alguno de los barcos en que viaja:

El navío viraba en bordadas muy largas, así que creímos que era mejor baquear que estar a la corda o correr a palo seco. Arrizamos el trinquete y lo largamos, y le halamos a popa la bolina; arronzábamos de mala manera. El navío trasorceaba de perlas. Encabillamos el cáñamo bajo de proa, pero la vela se rifó y arriamos la verga, y metimos la vela en cubierta y destrincamos todo hasta dejarla libre. Era una borrasca feroz (Swift, 2003: 276).

De este modo, ambos autores tratan de mostrar lo artificioso del lenguaje utilizado tanto en los libros de caballerías como en los libros de viajes, pues mientras el primer fragmento muestra que don Quijote se encuentra totalmente fuera de su época (incapacitado en ocasiones para ser comprendido por sus contemporáneos), la frialdad de Gulliver hace que sea casi necesario leer dos veces el segundo fragmento para darnos cuenta de lo trágica que es la situación que nos está narrando.

\subsection{Los tópicos}

Otro de los mecanismos al que ambos recurren es el de utilizar los tópicos que estaban presentes en esos géneros para ridiculizarlos. 
Así, igual que los libros de caballerías se presentaban como escritos por un antiguo cronista y el autor decía ser únicamente el traductor, en Don Quijote Cervantes dice que la obra la escribió el historiador arábigo Cide Hamete Benengeli tal y como hemos visto al hablar del juego de autoría. Aparte de este ejemplo, la inclusión de tópicos procedentes de los libros de caballerías en Don Quijote está bastante estudiada (vid. Marín Pina, 1998). Si hacemos un resumen de los más evidentes, podemos pensar en la investidura de don Quijote por el ventero en su primera salida, su firme determinación de defender a los menesterosos, la aventura del barco encantado, el descenso a la cueva de Montesinos o la mayoría de las escenas que preparan los duques para engañar al caballero y su escudero. En todos estos casos, la parodia funciona, a grandes rasgos, por la oposición entre el mundo real o extramundo y el mundo transformado por don Quijote a partir de su mundo interior. ${ }^{13}$ El protagonista detecta similitudes entre la realidad y lo que ha leído en los libros de caballerías, por lo que inmediatamente interpreta cada situación como si efectivamente fuese análoga a la que estaba presente en sus lecturas. El contraste entre el mundo real y el mundo transformado de don Quijote produce el efecto paródico buscado por Cervantes, sin que exista la necesidad de conocer realmente ningún libro de caballerías para apreciar la parodia.

Menos estudiadas están las relaciones entre Los Viajes de Gulliver y los libros de viajes. Sin embargo, se ha de tener en cuenta que los libros de viajes constituían un género bastante heterogéneo, de modo que su desarrollo dependía casi por completo del viaje real que había efectuado el autor. Por ello, los tópicos no son tan evidentes como en el caso de los libros de caballerías. A pesar de

${ }^{13}$ Utilizo la terminología de Juan Ignacio Ferreras, cuyo libro presenta un estudio bastante interesante sobre las formas de la parodia en Don Quijote (vid. Ferreras, 1982). 
esto, se pueden señalar como tópicos que Swift recoge de los libros de viajes: el hecho de incluir mapas de las zonas exploradas -en el caso de Swift, como es evidente, son mapas ficticios-; la presencia de pigmeos -si es que podemos considerar a los liliputienses como una sátira de las discusiones sobre esta tribu africana en los libros de viajes-; los capítulos enteros dedicados a relatar la historia del país, sus leyes, su política, etc.; la costumbre de los viajeros de llevar de vuelta a Inglaterra muestras de la flora y la fauna de los nuevos territorios descubiertos; la presencia de individuos de una increíble longevidad entre los habitantes de los territorios descubiertos, o el desarrollo del tópico del buen salvaje.

Por otro lado, a diferencia de lo que ocurre en Don Quijote, en Los Viajes de Gulliver el lector no posee la información sobre las características de los libros de viajes en la propia parodia, ya que el protagonista no imita a otros viajeros, cosa que caracteriza a don Quijote, sino que es realmente un viajero, sólo que viaja a países ficticios. Esta situación hace que para poder apreciar la parodia en toda su extensión sea necesario recurrir a fuentes externas al texto. De este modo, el efecto paródico no se produce por el contraste entre el mundo real y el mundo transformado en el mismo sentido que en Don Quijote, pues en el texto sólo se encuentra un mundo, el que relata Gulliver en primera persona y que pretende hacerse pasar por real. La parodia no está en la visión del mundo del personaje sino en la propia realidad que se presenta en el libro. ${ }^{14}$ El efecto paródico sólo se consigue entonces cuando se conoce el mundo relatado en los libros de viajes auténticos y se puede comparar con el mundo de Los Viajes de Gulliver.

${ }^{14}$ Una excepción sería el final de la obra, en el que Gulliver pierde la razón e interpreta el mundo desde una perspectiva excéntrica. En cualquier caso, hablaré de las similitudes entre las obras en lo referido al final de sus héroes más adelante. 


\subsection{Los ideales}

El quinto recurso utilizado por ambos autores es el de parodiar los ideales que defendían los libros de caballerías y los libros de viajes. En este caso es complejo desentrańar la postura que cualquiera de los dos autores poseían realmente con respecto a aquellos ideales -no es posible determinar hasta qué punto un autor se identifica con el mensaje que nos transmite, especialmente debido a los problemas de interpretación- pero sí que se puede defender que son parodiados por sus personajes. Así, al tratar de actuar en su época conforme a los antiguos ideales caballerescos, lo que don Quijote hace es parodiarlos, mostrando la inadecuación de sus ideas acerca de la justicia, del valor o del amor, al mundo real. Del mismo modo, Gulliver, al tratar de llevar su fe en el progreso y sus criterios de observación científica a los mundos de fantasía que visita, lo que provoca es la risa.

Es curioso el hecho de que si Don Quijote critica la pervivencia de ideales pasados en el presente y Los Viajes de Gulliver la fe en el progreso y el futuro, dos opciones totalmente contrapuestas, en ambos libros se recurra al viejo tópico de la historia como una degeneración progresiva ${ }^{15}$ que Jorge Manrique expresó de forma acertada con aquel "cualquiera tiempo pasado fue mejor". Sin embargo, esta aparente paradoja es comprensible porque Swift no pretendía lanzar un mensaje coherente y cohesionado a lo largo de todo el libro, y Gulliver cambia de personalidad de un momento a otro sin justificación narrativa alguna con el simple propósito de servir a Swift para satirizar aquello que le interesa en cada momento (Elena, 2003: 61). Así, mientras el tercer viaje es

${ }^{15}$ En Don Quijote es famoso el discurso sobre la edad de oro y la edad de hierro presente en el capítulo XI de la primera parte. En Los Viajes de Gulliver, el tópico se encuentra presente en varios lugares, pero se desarrolla especialmente en el cuarto viaje a través del tópico del buen salvaje en la figura de los houyhnhnm. 
la máxima expresión de crítica hacia la fe en el progreso -que ya estaba presente en los dos viajes anteriores-, el siguiente y último es una parodia del mito del buen salvaje, otro de los tópicos de los libros de viajes. Por otro lado, la crítica de Swift en estos aspectos responde también a los ideales de los libros de viajes, que a la vez que defendían el progreso, miraban con nostalgia a las tribus "primitivas" con las que entraban en contacto, desarrollando así el tópico del buen salvaje.

\subsection{El final de los héroes}

El último aspecto que quería comentar es la diferente posición en que terminan los protagonistas de las obras en relación con sus paródicas aventuras. De don Quijote sabemos que se volvió loco de tanto leer libros de caballerías, pero que tras volver a casa vencido por el Caballero de la Blanca Luna recupera la cordura y asegura tener el juicio "libre y claro, sin las sombras caliginosas de la ignorancia que sobre él me pusieron mi amarga y continua leyenda de los detestables libros de caballerías" (Cervantes Saavedra, 1998: 1217). Por su parte, Gulliver empieza su historia totalmente cuerdo, pero tras volver a Inglaterra después de su cuarto viaje decide asumir como propia la cultura de los houyhnhnm, los caballos parlantes. Siendo así, asegura pasar al menos cuatro horas diarias con dos caballos que ha comprado, tratando de imitar sus movimientos, mientras que rehúye el trato humano en la medida de lo posible, tapándose la nariz con hojas de ruda, espliego o tabaco para no soportar el repugnante olor a yahoo que despiden su mujer y sus hijos.

Como podemos ver, don Quijote y Gulliver sufren una transformación inversa pero simétrica. Mientras uno va de la locura a la cordura, el otro parte de la cordura para acabar loco. Sin embargo, el mensaje transmitido en ambos casos es similar. 
Mientras la vuelta de don Quijote a la cordura le sirve a Cervantes para que su personaje pueda condenar los libros de caballerías, reforzando con palabras el mensaje que llevaba transmitiendo con sus actos desde el principio de la obra, la locura final de Gulliver viene a criticar los libros de viajes al ridiculizarle por adoptar una cultura extrańa, primitiva, acorde con el mensaje moralizador que solían transmitir estas obras. La diferencia estriba en la explicitud con que Cervantes lanza su mensaje.

\section{Conclusiones}

A lo largo de las últimas páginas hemos podido observar cómo, a pesar de no poder asegurar una influencia de Don Quijote sobre Los Viajes de Gulliver, existen una gran cantidad de paralelismos en los mecanismos que ambos autores utilizan. Es posible que estos se deban a la propia naturaleza de la parodia literaria. Sin embargo, realizar la comparación entre estas dos obras no deja de ser interesante, puesto que poseen mucho más en común de lo que hasta ahora se ha venido reconociendo. No en vano, Swift, como la mayoría de sus contemporáneos, era un gran admirador de Cervantes, y a lo largo de su obra se pueden encontrar varias referencias explícitas a Don Quijote. Incluso en Los Viajes de Gulliver hemos podido ver cómo hay una escena que está inspirada en el prólogo de la segunda parte de la obra de Cervantes. Al mismo tiempo, la influencia de la obra de Cervantes en la Inglaterra del siglo XVIII fue inmensa, por lo que no es de extrañar que, sin ser consciente, Swift hubiera podido dejarse llevar por un cierto espíritu cervantino.

Para recapitular las principales semejanzas y diferencias que se han ido señalando a lo largo del análisis, se puede destacar el juego que tanto Swift como Cervantes establecen con respecto a la autoría del texto, muy similar dado que ninguno de los dos 
escritores reconoce su mano en la obra. En el mismo sentido, utilizan los prólogos para defenderse de las críticas que habían recibido, constituyéndose así en críticos literarios de su propia producción. Estos dos paralelismos están bastante vinculados a las circunstancias que rodearon la publicación de ambas obras, dado que el hecho de que Swift escondiera su autoría está estrechamente vinculado a la situación política de la época; del mismo modo, presumiblemente no habría existido un segundo prólogo a Los Viajes de Gulliver si el primer editor no hubiese modificado el texto. Sin embargo, como Hammond señala, una de las principales influencias de Cervantes sobre la novela inglesa del siglo XVIII es precisamente la integración de dudas epistemológicas acerca del carácter de la novela en la propia obra (Hammond, 1998: 262). Por ello, merece la pena considerar la posibilidad de una influencia cervantina a este respecto.

También, hemos podido ver cómo Cervantes y Swift utilizan a menudo dos recursos estilísticos para parodiar los libros de caballerías y los libros de viajes respectivamente: la utilización del lenguaje del género a parodiar con el fin de ridiculizarlo y el hecho de llevar al absurdo los tópicos de aquellos libros y criticar los ideales que defendían. Se trata de dos recursos que son utilizados de un modo general en la parodia. Sin embargo, de nuevo, una de las principales influencias cervantinas en la novela inglesa del XVIII es el tema de la colisión entre unos valores ideales derivados de un corpus literario y la vulgaridad de la vida cotidiana (Hammond, 1998: 262). Por todo esto, resulta pertinente preguntarse si también puede haber alguna muestra de influencia cervantina a este respecto.

Finalmente, hemos visto cómo la locura final de Gulliver le hermana en cierto sentido con don Quijote, que terminando su aventura recupera el juicio. Realiza así el personaje británico el camino inverso al del manchego pero con el mismo efecto: reforzar 
el mensaje de la parodia. Posiblemente, los mayores paralelismos entre la obra de Cervantes y la de Swift se encuentren en el final de Los Viajes de Gulliver. La locura del personaje y la relación que se establece entre las normas sociales y el mundo transformado que Gulliver tiene en su mente nos remiten no sólo al final de Don Quijote, sino también a su inicio. Y al ser el conflicto entre la locura y la ley, de nuevo en opinión de Hammond, una de las mayores influencias cervantinas en la novela inglesa del XVIII (Hammond, 1998: 262), sin duda la locura final de Gulliver debería analizarse desde este punto de vista.

\section{Bibliografía}

Bando Domínguez, Juana, 2007, "La huella cervantina en 'The Battle of the Books' de Jonathan Swift", en La huella de Cervantes $y$ del Quijote en la cultura anglosajona, Barrio J. M. y Crespo Allué, M. J. (eds.), Universidad de Valladolid, Valladolid.

Cervantes Saavedra, Miguel de, 1998, Don Quijote de la Mancha, Francisco Rico (ed.), Crítica, Barcelona.

Close, Anthony, 1998, "Las interpretaciones del Quijote", en Don Quijote de la Mancha, Francisco Rico (ed.), Crítica, Barcelona.

De Seta, Cesare, 1996, L'Italia del Grand Tour: da Montaigne a Goethe, Electa Napoli, Napoli.

Domínguez de Paz, Elisa y Pablo Carrascosa Miguel, 1990, "Swift, lector de Cervantes", en Actas del I Coloquio Internacional de la Asociación de Cervantistas, Anthropos, Barcelona.

Eisenberg, Daniel, 1995, La interpretación cervantina del Quijote, Compañía literaria, Madrid.

Elena, Pilar, 2003, “Introducción”, en Jonathan Swift, Los viajes de Gulliver, Cátedra, Madrid. 
Elias, A. C., 1998, "Swift's Don Quixote, Dunkin's Virgil Travesty, and Other New Intelligence: John Lyon's 'Materials for a Life of Dr. Swift,' 1765”, Swift Studies, vol. 13, pp. 27-104.

Ernst, Winifred, 2005, "Proportions of credulity and curiosity: Swift and Cervantes", Dieciocho: Hispanic Enlightenment, vol. 28, núm. 2, pp. 127-136.

Ferreras , Juan Ignacio, 1982, La estructura paródica del Quijote, Taurus, Madrid.

García Tortosa, Francisco, 1973, Viajes imaginarios en el siglo XVIII inglés y su fondo cultural, Universidad de Salamanca, Salamanca.

Garrido Ardila, Juan Antonio, 2014, Cervantes en Inglaterra: El Quijote y la novela inglesa del siglo XVIII, Centro de Estudios Cervantinos, Alcalá de Henares.

Guerra de Gloss, Terresa, 1988, "The humor in Cervantes and Swift”, Revista Canaria de Estudios Ingleses, núm. 16, pp. 213224.

Guerra Bosch, Teresa, 1999, "Jonathan Swift y El Quijote", en Actas del XXI Congreso Internacional AEDEAN, Secretariado de Publicaciones de la Universidad de Sevilla, Sevilla, pp. 121-126.

Guijarro Ceballos, Javier, 2007, El quijote cervantino y los libros de caballerias: calas en la poética caballeresca, Centro de Estudios Cervantinos, Alcalá de Henares.

Hammond, Brean, 1998, "Mid-Century English Quixotism and the Defence of the Novel", Eighteenth Century Fiction, vol. 10, núm. 3, pp. 247-268.

2009, "The Cervantic Legacy in the Eighteenth-Century Novel", en Juan Antonio Garrido Ardila, The Cervantean Heritage: Reception and Influence of Cervantes in Britain, Legenda, Oxford. 
Marín Pina, Mari Carmen, 1998, "Motivos y tópicos caballerescos”, en Don Quijote de la Mancha, Francisco Rico (ed.), Crítica, Barcelona.

Medina Casado, Carmelo, 2005, "Traducciones del Quijote al inglés y su influencia en la novela inglesa", en No ha mucho tiempo que vivía..., R. Alarcón Sierra (ed.), Universidad de Jaén, Jaén.

Pardo García, Pedro Javier, 2005, "El Quijote y la novela inglesa: de Laurence Sterne a James Joyce”, en La ficción novelesca en los Siglos de oro y la literatura europea, Ministerio de Educación y Ciencia, Madrid.

1995-1997, "Formas de imitación del Quijote en la novela inglesa del siglo XVIII: Joseph Andrews y Tristram Shandy", Anales cervantinos, núm. 33, pp. 33-164.

Paulson, Ronals, 1997, Don Quixote in England, Johns Hopkins University Press, Baltimore.

Randall, Dale B. J. y Jackson Campbell Boswell, 2009, Cervantes in Seventeenth-Century England, Oxford University Press, Oxford.

Roubaud, Sylvia, 1998, "Los libros de caballerías”, en Don Quijote de la Mancha. Fernando Rico (ed.), Crítica, Barcelona.

Swift, Jonathan, 2003, Los viajes de Gulliver, Cátedra, Madrid.

Torralbo Caballero, Juan de Dios y Cristina María Gámez Fernández, 2007, "La influencia de 'Viaje del Parnaso' en 'The Battle of the Books'", en La huella de Cervantes y del Quijote en la cultura anglosajona, Universidad de Valladolid, Valladolid.

Williamson, E., 1991, El Quijote y los libros de caballerías, Taurus, Madrid. 